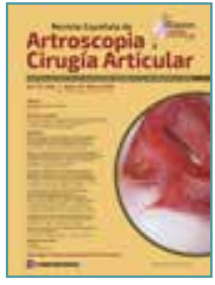

\title{
Originales
}

\section{Retorno al deporte posterior a una reconstrucción simultánea de ligamento cruzado anterior y ligamento colateral medial de grado IIII en periodo subagudo}

\author{
A. Bertona, J. P. Zicaro, J. M. González, N. Atala, C. Yacuzzi, M. Costa-Paz \\ Servicio de Ortopedia y Traumatología. Prof. Dr. Carlos E. Ottolenghi. \\ Hospital Italiano de Buenos Aires. Buenos Aires, Argentina
}

Correspondencia:

Dr. Bernardo Agustín Bertona Altieri

Correo electrónico: bernardo.bertona@hospitalitaliano.org.ar

\author{
Recibido el 16 de junio de 2017 \\ Aceptado el 4 de marzo de 2018 \\ Disponible en Internet: marzo de 2018
}

\section{RESUMEN}

Introducción: las lesiones asociadas del ligamento cruzado anterior (LCA) y del ligamento colateral medial (LCM) corresponden al $20 \%$ de la lesiones ligamentosas de la rodilla. El tratamiento conservador del LCM y la reconstrucción quirúrgica del LCA son generalmente recomendados. En deportistas, el tratamiento conservador del LCM puede conducir a inestabilidad medial crónica. El manejo óptimo de deportistas con lesiones combinadas de LCA-LCM continúa siendo controvertido. El objetivo de este trabajo es analizar los resultados funcionales y el nivel deportivo alcanzado en el postoperatorio de una serie de pacientes tratados con reconstrucción simultanea de LCA y LCM con un seguimiento mínimo de 2 años. Material y métodos: un total de 20 atletas con reconstrucciones agudas simultáneas de LCA y LCM de grado III fueron tratados entre marzo de 2006 y enero de 2014. El tiempo mínimo de seguimiento fue de 24 meses. Se evaluaron los resultados funcionales subjetivos (International Knee Documentation Committee -IKDC-, Lysholm), rango de movilidad, estabilidad anteromedial y rotacional (Lachmann, pivot shift, estrés valgo). Se registró la capacidad de retorno al deporte (Tegner) y el nivel alcanzado.

\begin{abstract}
Return to sports after sub-acute simultaneous reconstruction of anterior cruciate ligament injury and grade III medial collateral ligament injury
\end{abstract}

Introduction: combined anterior cruciate ligament (ACL) injury and medial collateral ligament (MCL) injury account for $20 \%$ of knee ligament lesions. Conservative treatment of $\mathrm{MCL}$ and surgical $\mathrm{ACL}$ reconstruction are generally recommended. Significant medial instability after non-surgical management of $M C L$ can lead to $A C L$ reconstruction failure. The optimal management for athletes with combined ACL-MCL injuries remains controversial. The purpose of this study was to analyze the functional results and the rate of return to sports of patients who underwent combined ACL-MCL surgery with minimum 2-years follow-up.

Methods: a total of 20 athletes with acute simultaneous ACL/grade III MCL reconstructions were treated between March 2006 and January 2014. The minimum follow-up time was 24 months. Subjective functional results (International Knee Documentation Committee -IKDC-, Lysholm), range of motion, anterior-medial and rotational stability (Lachmann, pivot shift, valgus stress) were evaluated. The ability to return to sport (Tegner) and the level achieved was recorded.

https://doi.org/10.24129/j.reaca.25161.fs1706021

FS $\odot 2018$ Fundación Española de Artroscopia. Publicado por Imaidea Interactiva en FONDOSCIENCE ${ }^{\circledR}$ (www.fondoscience.com) Este es un artículo Open Access bajo la licencia CC BY-NC-ND (www.creativecommons.org/licenses/by-nc-nd/4.0/). 
Resultados: todos los pacientes mejoraron significativamente en los scores funcionales y las maniobras de estabilidad. El IKDC preoperatorio promedio fue de 37,7 y el postoperatorio promedio fue de 88,21 . El Lysholm preoperatorio promedio fue de 40,44 y en el postoperatorio fue de 90,83. Todos los pacientes presentaron una movilidad normal/casi normal en el postoperatorio (IKDC A o B). Todos los pacientes retomaron la actividad deportiva. En el grupo de los deportistas de nivel competitivo, el 66\% alcanzó el mismo nivel deportivo que tenía previo a la lesión.

Conclusión: los pacientes con reconstrucción simultánea de LCA y LCM presentan a una mejoría significativa en los scores funcionales y las maniobras de inestabilidad de rodilla, logrando retomar su nivel deportivo previo en la mayoría de los casos.

Nivel de evidencia: IV, serie de casos.

Relevancia clínica: existen escasos reportes sobre el manejo de lesiones asociadas de LCA y LCM de grado 3 en deportistas. Los resultados de esta serie de casos son un aporte a la literatura. El manejo óptimo de este tipo de lesiones asociadas en deportistas continúa siendo un punto controvertido en la literatura.

Palabras clave: Ligamento cruzado anterior. LCA. Ligamento colateral medial. LCM. Retorno al deporte.

\section{Introducción}

El ligamento colateral medial (LCM) es la estructura ligamentosa de la rodilla más frecuentemente lesionada ${ }^{(1)}$. Anatómicamente se diferencia en LCM superficial (LCMS), LCM profundo (LCMp) y ligamento posterior oblicuo (LPO)(2).

Dependiendo del porcentaje de compromiso ligamentoso y la inestabilidad medial de la rodilla, las lesiones del LCM se dividen en los grados I, II y III, correspondiendo las lesiones de grado III a la disrupción completa de las fibras ligamentosas $^{(3)}$. Las lesiones de grado III están asociadas a otras lesiones ligamentosas en el $80 \%$ de los casos. De estas lesiones asociadas, el $95 \%$ corresponde a lesiones del ligamento cruzado anterior $(\mathrm{LCA})^{(4)}$. El mecanismo de lesión implica la carga en valgo de la rodilla, rotación externa o un vector de fuerza combinado que requiere la flexión de la rodilla ${ }^{(5-7)}$.
Results: all patients significantly improved functional scores and stability tests. The mean subjective IKDC score improved from $37.7 \pm 12.9$ (range: $21-69)$ preoperatively to $88.21 \pm 4.47$ (range: $80-96)$ postoperatively $(p<0.05)$. The average Lysholm score was $40.44 \pm 10.58$ (range: $27-65$ ) preoperatively and $90.83 \pm 3.38$ (range: $84-95$ ) postoperatively $(p<0.05)$. Valgus and sagittal laxity was not observed (IKDC A: 92\%; B: 8\%) at final follow-up. All patients had normal/nearly normal (IKDC A or B) mobility. All patients returned to sports. In the group of competitive athletes, $66 \%$ achieved the sports level prior to injury.

Conclusion: in athletes with acute ACL-grade III MCL lesions, an early simultaneous reconstruction can significantly improve the medial and sagittal stability of the knee. This procedure resulted in excellent functional outcomes, with return to the same level of sports in the majority of patients at short-term follow-up.

Level of evidence: IV, case series.

Clinical relevance: there is limited information on the management of associated ACL/MCL grade 3 injuries in athletes. The results of this series of cases are a contribution to the literature. The management of this type of associated injuries in athletes continuing being a controversial point in the literature.

Keywords: Anterior cruciate ligament. ACL. Medial collateral ligament. MCL. Return to sports.

El manejo de las lesiones de LCM depende del grado del compromiso ligamentoso. En lesiones asiladas de grado I y II el tratamiento no quirúrgico, con rehabilitación funcional precoz, es ampliamente aceptado. En lesiones de LCM de grado III, especialmente en asociación con lesión de LCA, el tratamiento es controvertido ${ }^{(8-12)}$.

Múltiples autores consideran que el tratamiento aislado del LCA en lesiones asociadas de LCA y LCM es suficiente para restaurar la estabilidad de la rodilla ${ }^{(13-15)}$. Sin embargo, existe evidencia de que en lesiones asociadas de LCA y LCM de grado III, la reparación aislada del LCA se relaciona con mayor carga mecánica sobre el LCA reconstruido, aumentando el riesgo de falla y de inestabilidad crónica sintomática en valgo de la rodilla ${ }^{(16-18)}$.

La reconstrucción simultánea de LCA y LCM en lesiones asociadas ha sido descrita con buenos resultados funcionales $(8,19,20)$. 
Los pacientes deportistas con lesiones asociadas de LCA y LCM de grado III son un grupo poco analizado en la literatura(21). Existe evidencia de que los pacientes con lesiones ligamentosas operados en el periodo agudo retoman su actividad deportiva de forma precoz, demostrando mejores resultados funcionales ${ }^{(22)}$.

El objetivo de este trabajo es analizar los resultados a corto plazo de pacientes deportistas con lesión asociada de LCA y LCM de grado III tratados en forma aguda con reconstrucción simultánea de ambas lesiones ligamentosas. Analizamos el retorno al deporte en el postoperatorio y las complicaciones. Nuestra hipótesis es que este tratamiento combinado mejora los resultados funcionales, especialmente en pacientes deportistas.

\section{Material y métodos}

Se realizó un análisis retrospectivo de pacientes deportistas intervenidos entre marzo de 2006 y enero de 2014 por lesiones asociadas de LCA y LCM de grado III agudas (menos de 6 semanas de evolución). El diagnóstico se realizó mediante examen físico y resonancia magnética nuclear objetivando disrupción completa de las fibras de ambas estructuras ligamentosas. Posterior al diagnóstico los pacientes fueron tratados con férula articulada y movilidad pasiva sin cargar peso durante 4 semanas. Al disminuir la inflamación aguda, los pacientes fueron evaluados nuevamente presentando en todos los casos inestabilidad rotacional, anteroposterior (Lachman, pivot shift), sumada a inestabilidad de grado III en valgo objetivada en radiografía con estrés en valgo a $20^{\circ}$, demostrando una diferencia con el lado contralateral mayor de $10 \mathrm{~mm}^{(23)}$. Fueron excluidos pacientes con otras lesiones asociadas (ligamento cruzado posterior, ligamento colateral lateral, etc.), pacientes con cirugías previas y pacientes que no fueran deportistas.

Todos los pacientes fueron operados por el mismo equipo quirúrgico, bajo anestesia raquídea y utilizando torniquete. Se realizó en primer término la reconstrucción del LCA en todos los casos, utilizando injerto autólogo de hueso-tendón rotuliano-hueso de la misma rodilla. El punto medio del túnel tibial en la superficie articular se localizó en promedio 2-3 mm anterior al borde

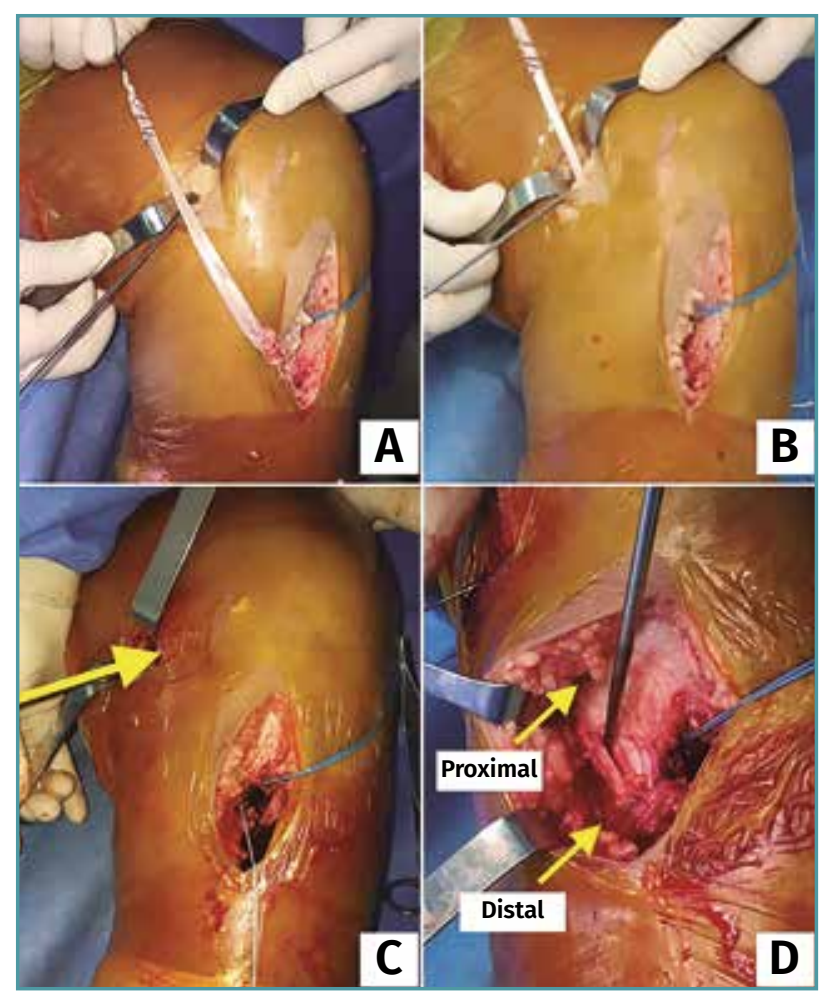

Figura 1. Reconstrucción del ligamento colateral medial. A: desinserción proximal de semitendinoso con extractor de isquiotiables abierto, manteniendo inserción distal; B: tunelización de semitendinoso; C: sitio de fijación femoral (grapa); D: sitio de fijación tibial. Distal a $6 \mathrm{~cm}$ de superficie articular (grapa). Proximal a $1,5 \mathrm{~cm}$ de superficie articular (sutura).

posterior del cuerno anterior del menisco externo y 7-8 $\mathrm{mm}$ anterior al ligamento cruzado posterior. El centro del túnel femoral se colocó en hora 10 para la rodilla derecha y en hora 2 para la rodilla izquierda, preservando $5 \mathrm{~mm}$ del borde posterior del cóndilo femoral; para ello, se colocó la rodilla a $120^{\circ}$ de flexión. La fijación tibial y femoral del LCA de reconstrucción se realizó mediante tornillos interferenciales metálicos. Posterior a la reconstrucción del LCA se realizó la reconstrucción del LCM. Se identificó el tendón del semitendinoso en su inserción tibial; mediante un extractor de isquiotibiales abierto, se desinsertó el semitendinoso de su inserción proximal. Para reproducir la inserción distal anatómica del LCMs se redireccionó a posterior el extremo distal del semitendinoso hacia el límite con el borde posterior de la tibia y se fijó a 5-6 cm de la superficie articular de la tibia mediante una grapa metálica de $5 \times 20 \mathrm{~mm}$. 
Posteriormente, el tendón fue tunelizado por debajo de la fascia del sartorio para alcanzar el sitio de inserción femoral descrito a 3,2 $\mathrm{mm}$ proximal y 4,8 mm posterior al epicóndilo medial(2). La fijación femoral se realizó mediante una grapa metálica de $5 \times 20 \mathrm{~mm}$. Finalmente, se realizó otro punto de anclaje reproduciendo la inserción proximal del LCMs a $12 \mathrm{~mm}$ de la superficie articular de la tibia, mediante un anclaje de 3,5 con suturas no reabsorbible (FiberWire ${ }^{\circledR}$ ). (Figura 1). No se reconstruyó el ligamento oblicuo posterior debido a que no presentaban inestabilidad rotacional posteromedial y a que se trató de casos subagudos.

Posterior a la cirugía, todos los pacientes utilizaron una férula articulada por 6 semanas, con ejercicios de rango de movilidad pasivos, fortalecimiento de cuádriceps desde el día 2 del postoperatorio. Se indicó no cargar peso durante 2 semanas, carga parcial con la utilización de muletas desde la semana 2 a la semana 5 y, posteriormente, carga completa. Todos los pacientes fueron incluidos en un programa de rehabilitación ambulatorio 3 veces por semana durante 8 semanas.

Se evaluaron los resultados funcionales mediante los scores subjetivos de IKDC (International Knee Documentation Committee) (24) y Lysholm (Lysholm Knee Scoring Scale) $)^{(25)}$. Se realizó una evaluación objetiva de la estabilidad y del rango de movilidad utilizando la escala objetiva del IKDC ${ }^{(26)}$. Se analizó la estabilidad rotacional y anteroposterior mediante las pruebas de Lachman (A: 0-2 mm; B: 3-5 mm; C: 6-10 mm; D: > $10 \mathrm{~mm}$ ) y pivot shift (A: normal; B: + leve; C: ++ marcado; D: +++ grosero). La estabilidad en valgo fue evaluada mediante la maniobra de bostezo en valgo (A: 0-2 mm; B: 3-5 mm; C: 6-10 mm; D: > $10 \mathrm{~mm}$ ). Se realizó una comparación de los valores del preoperatorio con los del último control postoperatorio. Se registró la capacidad de retornar al deporte (Tegner) y el nivel deportivo alcanzado en el postoperatorio. Se analizaron los datos utilizando el software STATA versión 13 (Stata Corporation, College Station, Texas, USA). Se consideró como estadísticamente significativo una $p<0,05$. El análisis fue aprobado por el comité de ética de nuestra institución.

\section{Resultados}

Veinte pacientes fueron incluidos. Ocho mujeres, 12 hombres, con una edad promedio de 22 años

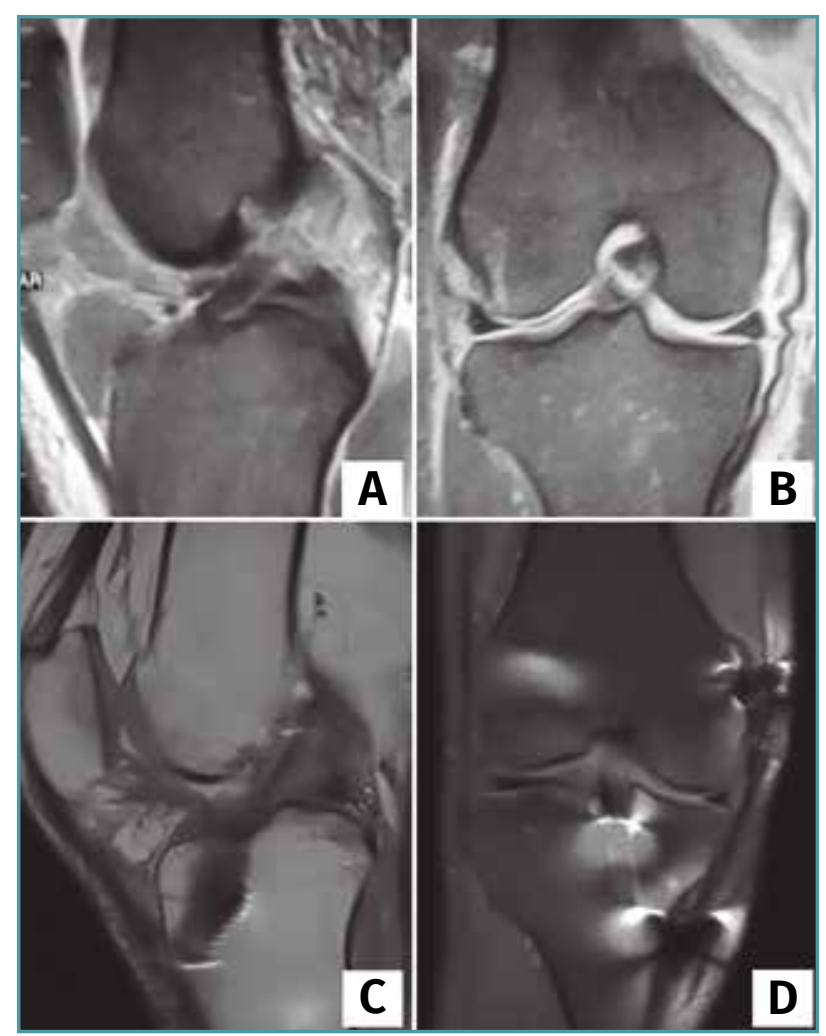

Figura 2. Resonancia magnética correspondiente a paciente de 23 años con lesión asociada de ligamento cruzado anterior (LCA) y ligamento colateral medial (LCM) de grado III. A y B: resonancia preoperatoria donde se observa lesión completa del LCA $(A)$ y lesión de LMC de grado III proximal (B).

(rango: 18-35 años). Once presentaron lesión en la rodilla derecha, nueve en la izquierda. El 85\% presentó compromiso del miembro inferior dominante. El seguimiento mínimo fue de 24 meses (promedio: 41 meses) (Figura 2).

El nivel deportivo previo a la lesión se analizó con la escala de Tegner, siendo 14 pacientes deportistas recreacionales (Tegner 5-7) y 6 pacientes deportistas de competición (Tegner 7-10).

Todos los pacientes presentaron una mejoría significativa en los scores funcionales y las maniobras de estabilidad en el último control postoperatorio. El score subjetivo del IKDC preoperatorio promedio fue de 37,7 $\pm 12,9$ (21-69) y el postoperatorio promedio fue de $88,21 \pm 4,47$ (80-96) ( $p<0,05)$. El score de Lysholm preoperatorio promedio fue de 40,44 $\pm 10,58(27-65)$ y en el postoperatorio fue de 90,83 $\pm 3,38$ (84-95) $(p<0,05)$. 
Tabla 1. Estabilidad

\begin{tabular}{c|c|c|c|c|c|c}
\hline & \multicolumn{2}{|c|}{ Pivot shift } & \multicolumn{2}{c|}{ Lachman } & \multicolumn{2}{c}{ Valgo (bostezo) } \\
\hline Grados IKDC* & Pre Op. & Post Op. & Pre Op. & Post Op. & Pre Op. & Post Op. \\
\hline A & 0,048 & $20(100)$ & & $18(90)$ & & $17(85)$ \\
\hline B & $3(15)$ & & & $2(10)$ & & $3(15)$ \\
\hline C & $10(50)$ & & $6(30)$ & & $4(20)$ & \\
\hline D & $7(35)$ & & $14(70)$ & & $16(80)$ & \\
\hline
\end{tabular}

IKDC: International Knee Documentation Committee

Resultados en número de pacientes y (porcentaje)

* Pivot shift A: normal B: + desplaza C: ++ marcado

* Lachman A: 0-2 mm B: 2-5 mm C: 6-10 mm

*Valgo (bostezo) A: 0-2 mm B: 2-5 mm C: 6-10 mm

C: +++ grosero

C: $>10 \mathrm{~mm}$

C: $>10 \mathrm{~mm}$

Del grupo de deportistas de competición (6/20 pacientes) (Tegner 7-10), 2 pacientes bajaron el nivel deportivo de competitivo a recreacional. Un paciente que realizaba rugby de manera competitiva (Tegner 9) cambió su deporte a ciclismo recreacional (Tegner 5), refiriendo temor a una nueva lesión. Un paciente que realizaba fútbol a nivel competitivo (Tegner 9) bajó su nivel deportivo a fútbol recreacional (Tegner 7) por

Tabla 2. Nivel deportivo

\begin{tabular}{c|c|c}
\hline Tegner* & Pre Op. & Post Op. \\
\hline 0 & & \\
\hline $1-4$ & & \\
\hline $5-7$ & $14(70)$ & $16(80)$ \\
\hline $7-10$ & $6(30)$ & $4(20)$ \\
\hline
\end{tabular}

Resultados en número de pacientes y (porcentaje)

* Tegner 5-7: deporte recreacional; 7-10: competitivo

Al analizar la estabilidad anteroposterior, rotacional y en valgo en el postoperatorio, mediante las pruebas de Lachman, pivot shift y estrés en valgo, todos los pacientes presentaron estabilidad normal (IKDC A: 92\%) o casi normal (IKDC B: 8\%) (Tabla 1).

El rango de movilidad en el último control postoperatorio fue normal/casi normal (IKDC A o B) (Tabla 1).

Todos los pacientes retomaron la actividad deportiva (20/20 pacientes) (Tabla 2) y el $90 \%$ alcanzó el nivel previo a la lesión ligamentosa. El tiempo promedio para alcanzar el máximo nivel deportivo en el postoperatorio fue de 17 meses (8-24 meses). Del grupo de deportistas recreacionales (14/20 pacientes), todos lograron retomar este nivel deportivo. Tres de estos pacientes cambiaron el tipo de deporte. Dos pacientes que realizaban fútbol de manera recreacional antes de la lesión (Tegner 7) cambiaron su deporte a maratón 2 veces por semana, alcanzando su nivel deportivo previo a la lesión (Tegner 7). motivos laborales. Dos futbolistas y dos jugadores de rugby retomaron su nivel competitivo previo (Tegner 9), siendo el $66 \%$ de este grupo (4/6 pacientes).

Tres pacientes presentaron complicaciones (15\%). Una paciente presentó rigidez postoperatoria; fue tratada con movilización bajo anestesia al tercer mes postoperatorio. Dos pacientes presentaron irritación en relación con la osteosíntesis a nivel femoral. Se realizó extracción de la grapa a los 26 y los 30 meses postoperatorios, respectivamente.

\section{Discusión}

El resultado principal de esta serie es que en pacientes deportistas con lesiones asociadas de LCA y LCM de grado III, la reconstrucción simultánea de ambas lesiones utilizando injertos autólogos de la misma rodilla se relacionó con buenos resultados funcionales y estabilidad. Todos los pacientes retomaron la actividad deportiva, logrando el $90 \%$ retomar el mismo nivel previo a la lesión.

La asociación de una lesión de LCA y LCM es la lesión multiligamentosa de la rodilla más frecuente $^{(1)}$. Si bien las lesiones aisladas del LCM tienen alto poder de cicatrización ${ }^{(27)}$, relacionándose con buenos resultados con tratamiento no quirúrgico ${ }^{(11,28)}$, el tratamiento de las lesiones completas del LCM (grado III) asociadas a lesión del LCA es un punto controvertido.

Existen autores que describen el tratamiento no quirúrgico de ambas lesiones como una op- 
ción aceptable $e^{(29,30)}$. Otros autores consideran que solo la reconstrucción aislada del LCA es suficiente para restaurar la estabilidad en lesiones asociadas de LCA y LCM de grado III (14,31). Así también, múltiples autores describen el tratamiento quirúrgico de ambas lesiones ligamentosas simultáneamente como tratamiento adecuado $(8,21,32,33)$.

Por otro lado, el momento de realizar la intervención quirúrgica, ya sea en agudo o diferido, también es un punto de controversia ${ }^{(7,14)}$. Grant et al. ${ }^{(13)}$, en una revisión sistemática, recomiendan tomar la decisión quirúrgica una vez pasado el periodo de inflamación aguda, habiendo recobrado el rango de movilidad completa. En caso de existir inestabilidad en valgo en este periodo, los autores sugieren realizar el tratamiento quirúrgico de ambas lesiones ligamentosas. En nuestra serie aplicamos un manejo similar al propuesto por este algoritmo, ya que consideramos fundamental evitar el dolor y la inestabilidad residual en valgo, que podrían presentar estos pacientes en caso de realizar aisladamente la reconstrucción del LCA.

La técnica quirúrgica a utilizar en la lesión

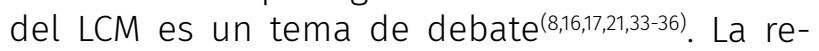
construcción del LCM utilizando injerto autólogo de isquiotibiales ha sido reportada con buenos resultados funcionales en pacientes con lesiones crónicas del LCM. Yoshiva et al. ${ }^{(20)}$ analizaron 12 pacientes a los que se les realizó una reconstrucción simultánea de ambas lesiones ligamentosas, utilizando injerto de semitendinoso autólogo para el LCM y tendón rotuliano para el LCA. Con un seguimiento mínimo de 2 años, los autores reportaron una movilidad normal/casi normal en todos sus pacientes, con una función normal/casi normal en el 92\% mediante la escala de IKDC.

En nuestra serie y en concordancia con publicaciones actuales, la reconstrucción de la lesión del LCM y del LCA se realizó en el periodo agu$\mathrm{do}^{(35,37)}$, teniendo en cuenta que el LCM durante el periodo agudo presenta un alto potencial de cicatrización, siendo esta característica utilizada como favorecedora para la integración de la reconstrucción ligamentosa, logrando la estabilidad en valgo en el postoperatorio.

Al analizar el retorno a la actividad deportiva es importante tener en cuenta que existen múltiples factores que influyen en este suceso independientemente de los buenos resultados funcionales: la edad del paciente, el nivel deportivo previo a la lesión, los miedos a una nueva lesión, motivos personales, laborales, familiares, entre otros; son factores que tienen una influencia determinante en el retorno al deporte y el nivel deportivo posterior a una cirugía ligamentosa. Tjong et al. ${ }^{(38)}$ analizaron mediante un cuestionario subjetivo a 31 pacientes, con buenos resultados funcionales (IKDC $A-B$ ), identificando que el miedo a presentar una nueva lesión y las prioridades personales son puntos fundamentales considerados por los pacientes a la hora de retornar al deporte. Ardern et al. ${ }^{(39)}$ analizaron 7.556 pacientes en una revisión sistemática donde incluyeron 69 artículos sobre retorno al deporte posterior a cirugía de reconstrucción del LCA. Reportaron que el $81 \%$ de los pacientes retorna al deporte y el 65\% logra el mismo nivel deportivo previo. Los autores describen que los principales factores relacionados con el retorno al deporte son el sexo masculino, la edad menor a 20 años, los deportistas de alto rendimiento y la actitud positiva. Osti et al. (21) evaluaron los resultados funcionales y la capacidad del retorno al deporte en 22 pacientes con lesión crónica de LCA/LCM, tratados con reconstrucción de LCA utilizando isquiotiables de la rodilla contralateral y reparación del LCM, reportando que el $92 \%$ de los pacientes lograron el mismo nivel deportivo previo a la lesión.

Si bien son resultados alentadores, cabe destacar que 5 de 20 pacientes (25\%) bajaron el nivel deportivo $(2 / 20)$ o cambiaron el deporte manteniendo el nivel deportivo (3/20).

La complicación más frecuente en la cirugía de reconstrucción del LCM es la artrofibrosis. Existen múltiples reportes que describen esta complicación, todos ellos en pacientes inmovilizados en extensión completa por un periodo promedio de 3 semanas en el postoperatorio con el objetivo de lograr cierto grado de cicatrización previo al inicio de la movilidad y prevenir la elongación de las fibras reconstruidas, evitando la inestabilidad residual ${ }^{(13)}$.

En nuestra serie, con el objetivo de evitar esta complicación, se estableció un protocolo de movilidad precoz, siguiendo lo reportado previamente en la literatura ${ }^{(33,36)}$. La complicación más frecuente en nuestros pacientes fue relacionada con la irritación producida por la osteosintesis a nivel del fémur distal; este análisis generó en los 
autores el cambio del sistema de fijación a nivel femoral en pacientes posteriores, no incluidos en esta serie por corto seguimiento.

Esta serie presenta como limitación principal el escaso número de pacientes y el no disponer de un grupo de comparación. Teniendo en cuenta la heterogeneidad de las series en la literatura en cuanto a la indicación quirúrgica, estrategia de reparación vs. reconstrucción a utilizar y el tiempo de realización de la intervención, el planteo de un grupo control se torna dificultoso. Otra limitación es que no se utilizaron herramientas de medición de estabilidad. Sin embargo, el hecho de que existe escasa información sobre el manejo de pacientes deportistas con lesiones asociadas del LCA y LCM de grado III le otorga relevancia a los datos obtenidos de esta serie.

\section{Conclusión}

En deportistas con lesiones agudas de LCA y LCM de grado 3, la reconstrucción simultánea de ambas lesiones en el periodo subagudo se asoció a una mejoría significativa de la estabilidad medial y sagital de la rodilla. Los pacientes presentaron excelentes resultados funcionales, logrando retomar el nivel deportivo previo a la lesión en el $90 \%$ en un análisis a corto plazo.

\section{Responsabilidades éticas}

Conflicto de interés. Los autores declaran no tener ningún conflicto de intereses.

Financiación. Este trabajo no ha sido financiado. Ninguno de los autores recibió financiación externa o subvenciones.

Protección de personas y animales. LOS autores declaran que los procedimientos seguidos se conformaron a las normas éticas del comité de experimentación humana responsable y de acuerdo con la Asociación Médica Mundial y la Declaración de Helsinki.

Confidencialidad de los datos. Los autores declaran que han seguido los protocolos de su centro de trabajo sobre la publicación de datos de pacientes.

Derecho a la privacidad y consentimiento informado. Los autores declaran que en este artículo no aparecen datos de pacientes.

\section{Bibliografía}

1. Miyasaka KC, Daniel DM, Stone ML HP. The incidence of knee ligament injuries in the general population. Am J Knee Surg. 1991;4:3-8.

2. LaPrade RF, Engebretsen AH, Ly TV, Johansen S, Wentorf FA, Engebretsen L. The anatomy of the medial part of the knee. J Bone Joint Surg Am. 2007;89(9):2000-10.

3. Hughston JC, Andrews JR, Cross MJ, Moschi A. Classification of knee ligament instabilities. Part I. The medial compartment and cruciate ligaments. J Bone Joint Surg Am. 1976;58(2):159-72.

4. Kovachevich R, Shah JP, Arens AM, Stuart MJ, Dahm DL, Levy BA. Operative management of the medial collateral ligament in the multi-ligament injured knee: an evidence-based systematic review. Knee Surg Sports Traumatol Arthrosc. 2009;17(7):823-9.

5. Warme WJ, Feagin JA, King P, Lambert KL, Cunningham RR. Ski injury statistics, 1982 to 1993, Jackson Hole Ski Resort. Am J Sports Med. 1995;23(5):597-600.

6. Lorentzon R, Wedrèn $H$, Pietilä T. Incidence, nature, and causes of ice hockey injuries. A three-year prospective study of a Swedish elite ice hockey team. Am I Sports Med. 1988; 16(4):392-6.

7. Peterson L, Junge A, Chomiak J, Graf-Baumann T, Dvorak J. Incidence of football injuries and complaints in different age groups and skill-level groups. Am J Sports Med. 2000;28(5 Suppl):S51-7.

8. Koga H, Muneta T, Yagishita K, Ju YJ, Sekiya I. Surgical management of grade 3 medial knee injuries combined with cruciate ligament injuries. Knee Surgery, Sport Traumatol Arthrosc. 2012;20(1):88-94.

9. Azar FM. Evaluation and treatment of chronic medial collateral ligament injuries of the knee. Sports Med Arthrosc. 2006;14(2):84-90.

10. Ballmer PM, Ballmer FT, Jakob RP. Reconstruction of the anterior cruciate ligament alone in the treatment of a combined instability with complete rupture of the medial collateral ligament. A prospective study. Arch Orthop Trauma Surg. 1991;110(3):139-41.

11. Battaglia MJ, Lenhoff MW, Ehteshami JR, Lyman S, Provencher MT, Wickiewicz TL, et al. Medial collateral ligament injuries and subsequent load on the anterior cruciate ligament: a biomechanical evaluation in a cadaveric model. Am J Sports Med. 2009;37(2): 305-11.

12. American Medical Association. Committee on the Medical Aspects of Sports. Standard nomenclature of athletic injuries. Chicago; 1966. pp. 99-100.

13. Grant JA, Tannenbaum E, Miller BS, Bedi A. Treatment of Combined Complete Tears of the Anterior Cruciate and 
Medial Collateral Ligaments. Arthrosc J Arthrosc Relat Surg. 2012 May 7;28(1):110-22.

14. Halinen J, Lindahl J, Hirvensalo E, Santavirta S. Operative and nonoperative treatments of medial collateral ligament rupture with early anterior cruciate ligament reconstruction: a prospective randomized study. Am J Sports Med. 2006;34(7):1134-40.

15. Hara K, Niga S, Ikeda H, Cho S, Muneta T. Isolated anterior cruciate ligament reconstruction in patients with chronic anterior cruciate ligament insufficiency combined with grade II valgus laxity. Am J Sports Med. 2008;36(2):333-9.

16. Liu X, Feng $H$, Zhang $H$, Hong L, Wang XS, Zhang J, et al. Surgical treatment of subacute and chronic valgus instability in multiligament-injured knees with superficial medial collateral ligament reconstruction using Achilles allografts: a quantitative analysis with a minimum 2-year follow-up. Am J Sports Med. 2013;41(5):1044-50.

17. Woo SL, Young EP, Ohland KJ, Marcin JP, Horibe S, Lin HC. The effects of transection of the anterior cruciate ligament on healing of the medial collateral ligament. A biomechanical study of the knee in dogs. J Bone Joint Surg Am. 1990;72(3):382-92.

18. Zaffagnini S, Bignozzi S, Martelli S, Lopomo N, Marcacci $M$. Does $A C L$ reconstruction restore knee stability in combined lesions?: an in vivo study. Clin Orthop Relat Res. 2007;454:95-9.

19. Zhang $H$, Sun $Y$, Han X, Wang $Y$, Wang L, Alquhali A, et al. Simultaneous Reconstruction of the Anterior Cruciate Ligament and Medial Collateral Ligament in Patients With Chronic ACL-MCL Lesions: A Minimum 2-Year Follow-up Study. Am J Sports Med. 2014;42(7):1675-81.

20. Yoshiya S, Kuroda R, Mizuno K, Yamamoto T, Kurosaka M. Medial collateral ligament reconstruction using autogenous hamstring tendons: technique and results in initial cases. Am J Sports Med. 2005;33(9): 1380-5.

21. Osti L, Papalia R, Del Buono A, Merlo F, Denaro V, Maffulli N. Simultaneous surgical management of chronic grade-2 valgus instability of the knee and anterior cruciate ligament deficiency in athletes. Knee Surg Sports Traumatol Arthrosc. 2010;18(3):312-6.

22. Marcacci M, Zaffagnini S, Iacono F, Neri MP, Petitto A. Early versus late reconstruction for anterior cruciate ligament rupture. Results after five years of followup. Am J Sports Med. 1995;23(6):690-3.

23. Wijdicks CA, Griffith CJ, Johansen S, Engebretsen L, LaPrade RF. Injuries to the medial collateral ligament and associated medial structures of the knee. J Bone Joint Surg Am. 2010;92(5):1266-80.
24. Anderson AF, Irrgang JJ, Kocher MS, Mann BJ, Harrast J); International Knee Documentation Committee. The International Knee Documentation Committee Subjective Knee Evaluation Form: normative data. Am J Sports Med. 2006;34(1):128-35.

25. Tegner $Y$, Lysholm J. Rating systems in the evaluation of knee ligament injuries. Clin Orthop Relat Res. 1985;(198):43-9.

26. Anderson A, Bergfeld J, Boland A, Dye S, Feagin J, Harner C, et al.; IKDC Committee AOSSM. IKDC Knee Forms. IKDC Committee AOSSM; 2000.

27. Nagineni CN, Amiel D, Green MH, Berchuck M, Akeson $\mathrm{WH}$. Characterization of the intrinsic properties of the anterior cruciate and medial collateral ligament cells: an in vitro cell culture study. J Orthop Res. 1992;10(4):465-75.

28. Fetto JF, Marshall JL. Medial collateral ligament injuries of the knee: a rationale for treatment. Clin Orthop Relat Res. 1978;(132):206-18.

29. Hillard-Sembell D, Daniel DM, Stone ML, Dobson BE, Fithian DC. Combined injuries of the anterior cruciate and medial collateral ligaments of the knee. Effect of treatment on stability and function of the joint. J Bone Joint Surg Am. 1996;78(2):169-76.

30. Jacobson KE, Chi FS. Evaluation and Treatment of Medial Collateral Ligament and Medial-sided Injuries of the Knee. Sports Med Arthrosc. 2006;14(2): 58-66.

31. Shelbourne KD, Porter DA. Anterior cruciate ligament-medial collateral ligament injury: nonoperative management of medial collateral ligament tears with anterior cruciate ligament reconstruction. A preliminary report. Am J Sports Med. 1992;20(3):283-6.

32. Marx RG, Hetsroni I. Surgical Technique: Medial Collateral Ligament Reconstruction Using Achilles Allograft for Combined Knee Ligament Injury. Clin Orthop Relat Res. 2012;470(3):798-805.

33. Lind M, Jakobsen BW, Lund B, Hansen MS, Abdallah O, Christiansen SE. Anatomical reconstruction of the medial collateral ligament and posteromedial corner of the knee in patients with chronic medial collateral ligament instability. Am J Sports Med. 2009;37(6): 1116-22.

34. Marchant MH, Tibor LM, Sekiya JK, Hardaker WT, Garrett WE, Taylor DC. Management of medial-sided knee injuries, part 1: medial collateral ligament. Am J Sports Med. 2011;39(5):1102-13.

35. Dong J, Wang XF, Men X, Zhu J, Walker GN, Zheng XZ, et al. Surgical Treatment of Acute Grade III Medial Collateral Ligament Injury Combined With Anterior Cruciate Ligament Injury: Anatomic Ligament Repair Ver- 
sus Triangular Ligament Reconstruction. Arthroscopy. 2015;31(6):1108-16.

36. Laprade RF, Wijdicks CA. Surgical technique: development of an anatomic medial knee reconstruction. Clin Orthop Relat Res. 2012;470(3):806-14.

37. Ateschrang A, Döbele S, Freude T, Stöckle U, Schröter $S$, Kraus TM. Acute MCL and ACL injuries: first results of minimal-invasive MCL ligament bracing with combined ACL single-bundle reconstruction. Arch Orthop Trauma Surg [Internet]. 2016;136(9):1265-72.
38. Tjong VK, Murnaghan ML, Nyhof-Young JM, Ogilvie-Harris DJ. A Qualitative Investigation of the Decision to Return to Sport After Anterior Cruciate Ligament Reconstruction. Am J Sports Med. 2014;42(2):336-42.

39. Ardern CL, Taylor NF, Feller JA, Webster KE. Fifty-five per cent return to competitive sport following anterior cruciate ligament reconstruction surgery: an updated systematic review and meta-analysis including aspects of physical functioning and contextual factors. $\mathrm{Br} J$ Sports Med. 2014;48(21):1543-52. 